\title{
Aprendizaje basado en proyectos colaborativos. Una experiencia con estudiantes del Grado en Geografía y Ordenación del Territorio Collaborative project-based learning. An experience with students of the Degree in Geography and Land Management
}

\author{
Juan M. Parreño-Castellano, Mercedes A. Rodríguez.Rodríguez \\ juan.parreno@ulpgc.es, mercedes.rodríguez@ulpgc.es \\ Departamento de Geografía, Grupo de Innovación Educativa GeoEduca \\ Universidad de Las Palmas de Gran Canaria (ULPGC) \\ Las Palmas de Gran Canaria, España
}

\begin{abstract}
Resumen- El aprendizaje basado en proyectos colaborativos (ABPC) es una metodología educativa innovadora que facilita el aprendizaje significativo y que incrementa la relación entre teoría y práctica. En este trabajo se presenta una experiencia educativa desarrollada en la asignatura Técnicas Cualitativas del Grado en Geografía y Ordenación del Territorio. Se concluye que el ABPC se ha mostrado como una metodología que ha facilitado la obtención de las competencias de la materia y de un aprendizaje contextualizado, ha dado coherencia temática y estructura a una asignatura que por su naturaleza pudiera parecer desestructurada, ha incrementado la motivación y convertido al alumnado en el principal artífice de su proceso de aprendizaje; ha estimulado la vocación investigadora y generado sentimientos de participación y relevancia. La práctica desarrollada destaca la importancia de la tutorización, de la evaluación continua, de la coordinación docente y de la temporalización de tareas como elementos cruciales para el éxito.
\end{abstract}

Palabras clave: aprendizaje autónomo; preguntas guía; evaluación continua; técnicas cualitativas; Geografía.

Abstract- Collaborative project-based learning (CPBL) is an innovative educational methodology that facilitates the meaningful learning and a greater relationship between theory and practice. This paper presents an educational experience developed in the Qualitative Techniques subject of the Degree in Geography and Land Management. It is concluded that CPBL is a methodology that facilitates the obtaining of competences and contextualized learning, gives thematic coherence and structure to a subject apparently unstructured, increases motivation, makes students be the main architects of their learning, stimulates the researching vocation and generates a feeling of participation and relevance. The practice developed highlights the importance of tutoring, continuous evaluation, teaching coordination and the timing of tasks as crucial elements for success.

Keywords: autonomous learning; driving question; continuous evaluation; qualitative techniques; Geography.

\section{INTRODUCCIÓN}

La enseñanza universitaria está viviendo un proceso de construcción o re-construcción de su identidad a partir de cambios internos y externos por el que pretende transformarse y adaptarse a las necesidades de la sociedad actual y del nuevo tipo de universitario que ocupa sus aulas.

No cabe duda de que el proceso de construcción del Espacio Europeo de Educación Superior (EEES) es un ejemplo de ello y que ha planteado (y plantea) grandes retos a la universidad española. Entre estos retos podemos mencionar la adopción de un nuevo sistema cíclico de títulos de grado y master, el desarrollo de criterios y sistemas de garantía y mejora de la calidad, la adopción del sistema europeo de créditos (ECTS) o la incorporación en los planes de estudio del desarrollo de habilidades transversales para complementar el aprendizaje de los contenidos (Garrigós y Valero, 2012). Pero, además, debemos añadir el desarrollo de modelos educativos innovadores en los que el estudiante deja de ser un ente pasivo y se convierte en artífice de su propio aprendizaje.

Los docentes comprometidos con el cambio han visto en el EEES una gran oportunidad para mejorar la calidad del proceso de enseñanza y aprendizaje mediante el uso de metodologías docentes activas y de sistemas de evaluación formativa, en contraposición a los modelos de enseñanza fuertemente basados en la lección magistral y la evaluación mediante uno (o pocos) exámenes.

Dentro de estas metodologías innovadoras que buscan un mayor protagonismo de los estudiantes encontramos el Aprendizaje Basado en Proyectos (en adelante ABP). Según Meneses (2013, p. 6) el "El ABP es un conjunto de tareas basadas en la resolución de preguntas o problemas a través de la implicación del alumno en procesos de investigación de manera relativamente autónoma que culmina con un producto final presentado ante los demás".

Al respecto, Travieso y Ortiz (2018, p. 126) plantean que "el ABP se descubre como una propuesta novedosa que ha alcanzado gran auge a nivel internacional (...), aun cuando surge en los años 60 es aplicado hoy día en numerosos niveles de enseñanza" y afirman que posee como sustento una teoría constructivista. Desde esta posición, se asume el conocimiento como una construcción del estudiante en su interacción con el entorno cercano donde reside y especialmente con las situaciones sociales a las que se enfrenta. 
En función de la bibliografía existente, el ABP presenta las siguientes características: 1) el proyecto estructura el aprendizaje y este debe ser significativo para las competencias de la asignatura y para la experiencia e intereses de los estudiantes; 2) el proceso parte de la necesidad y el deseo de aprender, 3) el aprendizaje debe conectar al alumnado con la realidad y propiciar el compromiso; 4) el proyecto se organiza a partir de la formulación de una pregunta guía, 5) el alumnado se organiza en pequeños grupos autodirigidos que debe buscar información, debatir contenidos y tomar decisiones en el proceso investigador; 6) el profesorado debe ser facilitador o tutor en todas las fases, 7) el proyecto se presenta a una audiencia externa al grupo y 8) el alumnado debe ser evaluado por los otros estudiantes y por el profesor a partir de la formulación previa de rúbricas (Vergara Ramírez, 2016).

Con el ABP se puede estimular el Aprendizaje Colaborativo (AC) en la que medida en que requiere de la participación de pequeños grupos. El Aprendizaje Basado en Proyectos Colaborativos (ABPC) es diferente al basado en el trabajo cooperativo (Johnson y Johnson, 1999) ya que los estudiantes son capaces de tomar decisiones consensuadas, repartirse las tareas, aprender mediante la interacción de iguales y asumir el compromiso sobre la totalidad del proyecto dentro del grupo de una manera autogestionada (Bould, Cohen y Sampson, 2013; Cardozo, 2010; Guerra Santana, Rodríguez Pulido y Artiles Rodríguez, 2019, Maldonado, 2018).

En última instancia, el ABPC pretende generar una experiencia educativa en el alumnado que permita convertir los contenidos en herramientas que puedan ser usadas en otros contextos y que implique las dimensiones racional, relacional o emocional en el proceso de aprendizaje. La experiencia educativa se produce cuando el aprendizaje ha permitido un cambio en la persona que lo realiza a nivel cognitivo, físico, emocional o de compromiso social (Pérez Gómez, 2012).

\section{CONTEXTO}

El presente trabajo aborda una experiencia didáctica desarrollada en un aula de la Universidad de Las Palmas de Gran Canaria (ULPGC) en la que se muestra que el ABPC es una herramienta eficaz para la producción de conocimientos en estudiantes universitarios. El objetivo del presente trabajo es describir y analizar la experiencia y conocer la percepción que han tenido los propios estudiantes sobre el proceso educativo.

La experiencia se desarrolla en la asignatura de Técnicas Cualitativas del Grado en Geografía y Ordenación del Territorio. Se trata de una asignatura de formación obligatoria, impartida en tercer curso, que incluye 6 créditos ECTS (60 horas) presenciales y 90 horas de aprendizaje no presencial. La asignatura no cuenta con ningún antecedente en los cursos anteriores en los que los conocimientos metodológicos sobre cartografía, tecnologías de la información geográfica, bases de datos y estadística son preponderantes. A ello se suma que el alumnado apenas ha recibido formación sobre técnicas cualitativas en niveles educativos previos.

La necesidad de desarrollar un método didáctico alternativo en la asignatura parte de dos razones fundamentales que menoscababa la motivación para el aprendizaje y los resultados obtenidos. En primer lugar, la asignatura arrastraba un cierto estigma que se apreciaba en la valoración del alumnado. Al incluir técnicas en las que tienen cabida componentes subjetivos del conocimiento, el alumnado tenía la percepción de que la asignatura era menos rigurosa y, por tanto, de escaso valor en relación con otras materias que se percibían como objetivas. Y, en segundo lugar, el nivel de satisfacción del alumnado estaba por debajo del nivel medio de la titulación debido, sobre todo, al estudio apresurado de un elevado número de técnicas de análisis y a la falta de una estructura clara en la materia.

Por tanto, para lograr una mejora en la motivación y en los resultados académicos, había que abordar el reto de valorizar y dar coherencia a los contenidos. Ya que no se podía plantear la adaptación curricular de competencias, objetivos o contenidos, que están determinados en la memoria de verificación del título, se optó por un cambio metodológico que primara la actividad práctica y la relación de las diferentes unidades didácticas

Con este doble propósito se optó por una metodología de ABPC que nos permitiese desarrollar un planteamiento didáctico basado en la práctica y que vinculase todas las unidades de contenido a través de la ejecución del proyecto. Pero, además, esta herramienta de aprendizaje permitía que el alumnado consiguiera un aprendizaje significativo y duradero en el tiempo y llegara a tener una experiencia educativa multifacética que incluyera aspectos cognitivos, emocionales y relacionales. En relación con lo primero, baste mencionar que, según el cono de aprendizaje de E. Dale se recuerda el $90 \%$ de lo que decimos y hacemos diez días después de haberlo dicho o hecho.

Debemos indicar que se optó por el aprendizaje colaborativo y no por el cooperativo porque se pretendía que los estudiantes fueran los que diseñaran su estructura de interacción y los principales responsables en la toma de decisiones en el proceso. En un proyecto cooperativo el profesorado tiene un nivel de control mayor, algo que se quería evitar (Bruffee, 1999; Delgado, 2016, Monedero y Durán, 2002). Por otro lado, la dimensión colaborativa encajaba mejor en un planteamiento didáctico que pretendía favorecer el proceso en detrimento del resultado, ya que este podía verse afectado por factores de contexto a lo largo del semestre (Barkley, Cross y Major, 2007).

\section{DESCRIPCIÓN}

El planteamiento didáctico basado en $\mathrm{ABPC}$ de la asignatura Técnicas Cualitativas se ha desarrollado en los dos últimos cursos académicos. Previamente, se llevó a cabo un pilotaje durante los dos cursos anteriores en los que se aplicó el ABP en un entorno cooperativo, primero, y colaborativo, después, en determinadas unidades temáticas. Los resultados de esta fase previa de preparación posibilitaron implantar el ABPC como elemento didáctico estructurante de la totalidad de la asignatura. La descripción que sigue está referida al curso, 2020-21.

La metodología didáctica se extiende a lo largo de las quince semanas del semestre. Al comienzo del mismo, se le plantea al alumnado la realización de un proyecto colaborativo el que tendrán que aplicar las técnicas y herramientas que se irán enseñando en la parte teórica de la asignatura. Se informa y se discute en clase el proyecto docente de la asignatura, los objetivos y competencias previstos, las diferentes etapas que conforman el proyecto, lo que supone un planteamiento colaborativo, el nivel de compromiso que requiere y el método de evaluación. Esta fase de presentación es de gran importancia ya que sin entendimiento y compromiso inicial del alumnado difícilmente se obtendrán resultados satisfactorios. 
Los estudiantes constituyen los diferentes grupos y escogen la temática del proyecto en función de sus intereses personales. Esta debe estar relacionada con una problemática que les afecta directa o indirectamente o con la realidad social existente en su entorno geográfico. Dado que la asignatura se desarrolla en el ámbito de la Geografía, la temática debe vincularse a esta disciplina. La selección final de los temas se realiza tras el diálogo y el acuerdo de los miembros del grupo con la participación del profesorado de la asignatura. En algunos casos, el profesorado sugiere temas específicos relacionados con los temas de investigación del Departamento o con la problemática geográfica de actualidad e invita a encontrar las relaciones que puedan tener estos temas con el ámbito geográfico cercano a los estudiantes, con el propósito de estimular el compromiso con el trabajo y garantizar un mejor resultado. En todo este proceso, el profesorado actúa como un elemento evaluador y de apoyo, evitando siempre influir en la toma de decisiones.

En el caso del desarrollo educativo del último curso académico, la clase se dividió en cinco equipos de tres miembros y los temas seleccionados fueron:

- $\quad$ Equipo 1: Teleeducación durante la crisis pandémica.

- Equipo 2: Caracterización de un nuevo perfil de usuario de los espacios naturales a consecuencia de la pandemia de COVID-19: implicaciones, tendencias y prospecciones.

- $\quad$ Equipo 3: Percepción social sobre la problemática del deterioro del sistema dunar de Maspalomas y posible mejora debido al confinamiento.

- $\quad$ Equipo 4: Los problemas de la accesibilidad para personas con movilidad reducida en los espacios urbanos y rurales.

\section{- $\quad$ Equipo 5: Inmigración irregular en xxx.}

Como se puede apreciar, los tres primeros temas están directamente relacionados con la situación de crisis sanitaria que se estaba viviendo y, más específicamente, con la situación de confinamiento de unos meses antes, proponiéndose el análisis de las repercusiones que esto ha tenido en la sostenibilidad de dos tipos de espacios geográficos (un sistema dunar y un espacio natural protegido de montaña) o en el propio desempeño educativo del estudiante. Las otras dos temáticas, centradas en las personas con movilidad reducida y la inmigración irregular, se vinculaban con intereses del alumnado, ya que algunos estudiantes eran miembros de asociaciones o desempeñaban funciones de voluntariado orientadas a la inserción de personas con movilidad reducida y a la acogida de inmigrantes llegados irregularmente.

Tras esta etapa inicial orientada a la información, organización y motivación, el proyecto se organizó en seis fases (tabla 1). La primera fase se orientó a que los grupos conocieran diferentes puntos de vista en relación con el tema en el que iban a trabajar a partir de la consulta de bibliografía y recursos electrónicos, de tal manera que pudieran definir de una forma más adecuada objetivos precisos del trabajo e incluso apuntaran hipótesis de análisis. En otros términos, en esta fase se formularon las preguntas guías.
Tabla 1. Fases del modelo de aprendizaje basado en proyectos colaborativos.

\begin{tabular}{|c|c|c|}
\hline & Descripción & Observaciones \\
\hline 1 & $\begin{array}{l}\text { Después de la selección del } \\
\text { tema: } \\
\text { - Búsqueda bibliográfica y } \\
\text { elaboración del marco } \\
\text { teórico del tema. } \\
\text { - Justificación del interés } \\
\text { por el tema a investigar. } \\
\text { - Determinación de la zona } \\
\text { de estudio y el objetivo del } \\
\text { proyecto (preguntas guía). }\end{array}$ & \multirow{5}{*}{$\begin{array}{l}\text { El desarrollo de las } \\
\text { diferentes fases fue } \\
\text { monitoreado } \\
\text { facilitado por el } \\
\text { profesorado de la } \\
\text { asignatura durante las } \\
\text { sesiones de actividades } \\
\text { prácticas presenciales. } \\
\text { Así mismo, a medida } \\
\text { que avanzaba el } \\
\text { proyecto, los grupos de } \\
\text { trabajo debían elaborar } \\
\text { unos materiales que } \\
\text { tenían carácter } \\
\text { evaluativo. } \\
\text { De esta forma, ninguno } \\
\text { de los grupos se } \\
\text { quedaba descolgado y } \\
\text { se garantizaba un } \\
\text { trabajo continuado }\end{array}$} \\
\hline 2 & $\begin{array}{l}\text { - Diseño de una entrevista. } \\
\text { - Selección de un experto a } \\
\text { entrevistar. } \\
\text { - Trascripción y análisis de } \\
\text { la entrevista. }\end{array}$ & \\
\hline 3 & $\begin{array}{l}\text { - Diseño de una encuesta. } \\
\text { - Realización de la } \\
\text { encuesta. } \\
\text { - Análisis de la encuesta. }\end{array}$ & \\
\hline 4 & $\begin{array}{l}\text { - Preparación y realización } \\
\text { de un grupo de discusión } \\
\text { por proyecto. } \\
\text { - Transcripción y análisis } \\
\text { del grupo de discusión. }\end{array}$ & \\
\hline 5 & $\begin{array}{l}\text { - Realización de un análisis } \\
\text { DAFO. } \\
\text { - Discusión de estrategias a } \\
\text { partir del análisis DAFO. }\end{array}$ & \\
\hline 6 & $\begin{array}{l}\text { - Diseño y elaboración de } \\
\text { un póster por equipo. } \\
\text { - Presentación oral y } \\
\text { defensa de los proyectos. }\end{array}$ & $\begin{array}{l}\text { Esta fase tuvo dos } \\
\text { etapas: una, en el aula, } \\
\text { durante las sesiones de } \\
\text { prácticas y las tutorías } \\
\text { con los diferentes } \\
\text { grupos y, otra, de } \\
\text { presentación de los } \\
\text { resultados en sesión } \\
\text { plenaria, en la que el } \\
\text { profesorado de la } \\
\text { asignatura ejerció de } \\
\text { tribunal y cada } \\
\text { miembro de la clase } \\
\text { valoró el trabajo de sus } \\
\text { compañeros. }\end{array}$ \\
\hline
\end{tabular}

Las cuatro siguientes fases se centraron en una técnica específica. Los desarrollos de las mismas estaban en correspondencia con los temas tratados en las clases teóricas, por lo que los estudiantes aprendían haciendo mediante su investigación cada una de las diferentes técnicas. De esta manera, los grupos tuvieron que 1) seleccionar un informante cualificado, preparar un cuestionario de entrevista semiestructurada y realizar y transcribir la entrevista; 2) preparar un cuestionario para la realización de una encuesta cualitativa, seleccionar la muestra y obtener y tratar la información; 3) diseñar un grupo de discusión sobre la temática del trabajo y transcribir los resultados y 4) realizar un análisis 
DAFO final a tenor de toda la información y discusión que había generado el proyecto.

Las actividades se realizaron de manera presencial, usando un aula de informática o el aula de clase, y de modo no presencial. El uso del aula virtual y, en general, de las TIC en la asignatura fue de gran utilidad para facilitar la coordinación y el cumplimiento de la periodización prevista. En todas las fases el profesorado actúa como un facilitador, aportando recursos y ayudando a buscar soluciones. De la fase segunda a la quinta se combinó el método con un trabajo de enseñanza de transmisión de contenido de las técnicas como paso previo a su asimilación a través de la práctica.

La última fase del proyecto implicó la realización y presentación ante la clase de un poster en el que se sintetizaba el trabajo desarrollado y los principales resultados (figura 1). Esta última fase implicó también la tutorización de las actividades presenciales y culminó la evaluación continua. Los estudiantes expusieron cómo habían desarrollado el proyecto, los pasos que dieron, los problemas a los que tuvieron que hacer frente y cómo los habían solucionado y los principales resultados obtenidos. La presentación del póster a la clase permitió que cada grupo fuera evaluado por el resto del alumnado de manera cualitativa, sin que esto influyera en la calificación. Los estudiantes, generalmente, centraron su intervención en el proceso más que en el resultado, preguntando sobre las dificultades encontradas y la toma de decisiones realizadas.

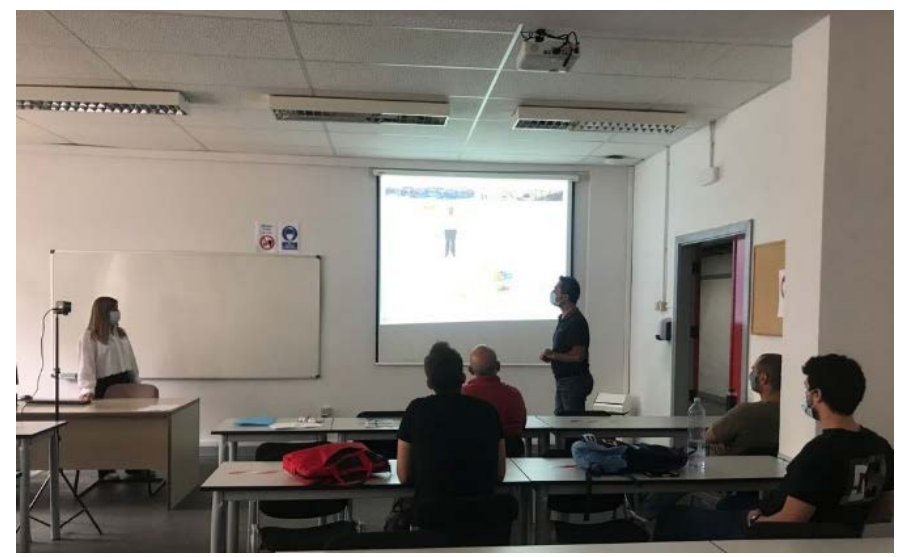

Figura 1. Exposición de los resultados en sesión plenaria. Fuente: Elaboración propia

La evaluación fue en todo momento formativa, basada en la elaboración de un portafolio de tareas y en el diseño de rúbricas (Bordas y Cabrera, 2001). Se centró tanto en el proceso, en el modo en que se habían solucionado las dificultades y en la toma de decisiones, como en los resultados específicos en cada tarea. En relación con la evaluación del proceso, dado que el alumnado trabajaba en grupo, se tuvo que hacer durante todo el tiempo de clase mediante la observación de los diferentes grupos. Para que esta evaluación fuera formativa se trasladaba al alumnado de manera recurrente.

En relación con los resultados, se evaluó sobre todo cómo la tarea práctica reflejaba la asimilación de los principios teóricos de la técnica, indistintamente de los resultados específicos de su aplicación. Por ejemplo, en el caso de la elaboración del cuestionario de la entrevista semiestructurada, se evaluó cómo esta respondía a los principios teóricos de toda entrevista y no tanto a cómo se había ejecutado. Esta evaluación se realizó a partir de las tareas del portafolio usando rúbricas específicas que el alumnado conocía previamente. El carácter formativo obligaba a cierta inmediatez en el proceso evaluador, dado que algunas competencias se evaluaban de manera reiterada a través de diferentes tareas. Con el portafolio, la evaluación se tradujo en calificación, reflejando tanto el resultado como el proceso, con el propósito de que se convirtiera en un estimulador de reflexión en el grupo.

Una vez concluido el proyecto, se solicitó al alumnado que respondiera un cuestionario realizado ad hoc $y$ autoadministrado a través del aula virtual de la asignatura, compuesto por las siguientes preguntas, además de algunos datos personales, con el fin de evaluar los logros de la herramienta de aprendizaje.

1) ¿Consideras que la metodología del trabajo por proyectos de la asignatura te ha ayudado a aprender sobre las técnicas cualitativas?

2) La forma de realizar las actividades prácticas, ¿te ha motivado para el aprendizaje de la asignatura?

3) Indica tu nivel de satisfacción con la metodología utilizada en la asignatura de Técnicas Cualitativas

4) ¿Recomendarías que esta metodología empleada se vuelva a utilizar en curso venideros?

5) Sugerencias. Aspectos positivos y/o aspectos negativos a señalar

Las tres primeras preguntas se respondían mediante una escala Likert de cinco grados en la que 1 indicada el total desacuerdo y 5 el acuerdo completo. La cuarta pregunta era cerrada de opción múltiple y la quinta era abierta. Las respuestas permitieron reflexionar sobre la actividad desarrollada y sobre las posibles prácticas de mejora.

Se veló porque todo el procedimiento cumpliera con los valores y prácticas éticas requeridas dentro de la investigación educativa: consentimiento voluntario informado, derecho a la información, protección de datos y garantías de confidencialidad, anonimato y no discriminación.

\section{Resultados}

Con la aplicación de la metodología ABPC se contribuyó al desarrollo de diferentes competencias, tanto generales como específicas, de la titulación y la asignatura. Entre las primeras la orientación didáctica incide directamente en estimular el conocimiento y capacidad de aplicación del método científico y la capacidad de análisis y síntesis, la resolución de problemas, la toma de decisiones, el trabajo en equipo, la gestión de la información y la posibilidad de aplicar los conocimientos teóricos en la práctica. Además, tiene una clara incidencia en el desarrollo del aprendizaje autónomo y en el conocimiento y la sensibilización hacia los derechos humanos y la reducción de todo tipo de desigualdad.

Entre las específicas, el planteamiento didáctico se ha mostrado eficaz en la obtención de algunas competencias como el conocimiento de la geografía humana, económica y social, la 
capacidad de utilizar la información geográfica como instrumento de interpretación del territorio, la de combinar las dimensiones temporal y espacial en la explicación de los procesos socioterritoriales o la de relacionar y sintetizar información territorial transversal. El carácter activo y colaborativo del ABPC supone conseguir otras metas formativas que no se expresan directamente a través de competencias u objetivos como el desarrollo de la autoestima, el autorreconocimiento o las habilidades de liderazgo. Estas competencias no están formuladas en el currículo de la asignatura, pero fueron claramente reconocibles. Es habitual en ABPC que esto ocurra (Vergara Ramírez, 2016).

Asimismo, el desarrollo de competencias transversales se relaciona con esta metodología aplicada al posibilitar al estudiante comunicarse con diferentes audiencias, utilizar los soportes y vías de comunicación especialmente relacionados con las nuevas tecnologías de la información y la comunicación, y permitir con sus competencias y conocimientos profesionales, la satisfacción de los intereses, necesidades y preocupaciones de su territorio.

Los estudiantes mostraron en todo momento su motivación por las actividades y por la utilización de las diferentes técnicas cualitativas en su investigación, lograron un aprendizaje contextualizado y significativo de los contenidos de la materia. Esto se corroboró a través del cuestionario autoadministrado. En la Tabla 2 se muestran las medias y desviaciones típicas de los ítems evaluados con escala de Likert. Tal y como se puede apreciar, los dos ítems con mayor puntuación media y menor desviación típica son los relacionados con el nivel de satisfacción con la metodología utilizada y con el proceso de aprendizaje. No sólo la valoración del aprendizaje, la motivación y la satisfacción es alta, sino que se registra homogeneidad en las opiniones, con desviaciones típicas reducidas y tasas de variación entre el 11 y $15 \%$. Esto pone en valor la práctica colaborativa, ya que los estudiantes en su conjunto se han visto concernidos.

Tabla 2. Resultados de la evaluación de la metodología realizada por el estudiantado

\begin{tabular}{|l|c|c|}
\hline \multicolumn{1}{|c|}{ Item } & Media & $\begin{array}{c}\text { Desviación } \\
\text { Típica }\end{array}$ \\
\hline $\begin{array}{l}\text { ¿Consideras que la metodología del } \\
\text { trabajo por proyectos de la } \\
\text { asignatura te ha ayudado a aprender } \\
\text { sobre las técnicas cualitativas? }\end{array}$ & 4,4 & 0,49 \\
\hline $\begin{array}{l}\text { La forma de realizar las actividades } \\
\text { prácticas, ite ha motivado para el } \\
\text { aprendizaje de la asignatura? }\end{array}$ & 4,1 & 0,64 \\
\hline $\begin{array}{l}\text { Indica tu nivel de satisfacción con la } \\
\text { metodología utilizada en la } \\
\text { asignatura de técnicas cualitativas }\end{array}$ & 4,5 & 0,63 \\
\hline
\end{tabular}

El hecho de que el alumnado recomiende la utilización de esta metodología en futuros cursos es el mejor indicador de aprendizaje y motivación. Los aspectos más valorados son el carácter práctico de la metodología, la combinación de actividades presenciales con no presenciales, la selección de temas y la relación con su experiencia, la diversidad de técnicas y temáticas abordadas, el estilo evaluador y la puesta en práctica de un sistema de evaluación continua y el desarrollo de inquietudes investigadoras. Las recomendaciones de mejora que realizaron los estudiantes se han centrado en la periodización de las actividades, la coordinación docente y la introducción de software específico de tratamiento de datos cualitativos.

\section{CONCLUSIONES}

Los cambios que se están produciendo en la sociedad y en la educación universitaria exigen la innovación en el proceso educativo con el fin de que el alumnado logre objetivos y competencias de una manera óptima y útil para la puesta en práctica del conocimiento. En el caso analizado, la falta de formación previa sobre los contenidos de la asignatura y la amplitud temática y de competencias presentes en el proyecto docente hacían complicado que, en un semestre, los resultados pudieran ser satisfactorios en un semestre.

Por ello, teniendo en cuenta la amplitud del grupo de clase, se optó por una metodología basada en proyectos desarrollada en un entorno colaborativo. Este planteamiento convertía al estudiante en el elemento activo de su aprendizaje, le permitía desarrollar diferentes tipos de competencias, estimulaba la motivación por el aprendizaje y daba coherencia interna a la asignatura en su estructuración teórica, en la relación entre teoría y práctica y en el propio proceso de enseñanzaaprendizaje.

Además, el cambio social de los últimos años, nos está llevando a un escenario en el que cada vez es más necesario que no solo los estudiantes aprendan contenidos, sino que aprendan a utilizarlos y a contextualizarlos en el territorio. En este sentido el ABPC es una metodología diseñada para la aplicación práctica real de los aprendido y la obtención de un aprendizaje significativo y contextualizado.

La clave para que este planteamiento metodológico sea exitoso estriba en siete $\mathrm{u}$ ocho elementos esenciales (Larmer y Mergendoller, 2010): la elección de contenido significativo, la buena formulación de preguntas guía, el estímulo de la necesidad de saber, la autonomía en el aprendizaje y la capacidad de decisión del alumnado en el proceso, la evaluación formativa y continua, el uso de tecnologías propias de nuestro tiempo, la formulación de competencias adecuadas y la presentación final ante la clase o la ciudadanía.

Teniendo en cuenta la experiencia desarrollada, queremos destacar algunos aspectos esenciales que debieran tenerse en cuenta para su aplicación en otros contextos: 1) la comprensión por todas las partes de las implicaciones que conlleva un proceso colaborativo en el que cada cual aporta habilidades diferenciadas que deben ser reconocidas. Una de las claves de éxito es que el alumnado debe entender qué es un ABPC y cómo va a ser evaluado; 2) la aceptación de roles diferenciados por parte de los estudiantes y del profesorado. Los primeros deben asumir su papel central en su propio aprendizaje y los segundos deben relegar su papel de transmisor de contenidos por el de tutela continua; 3) la fuerte relación que debe haber entre la 
temática abordada y la experiencia vital o los intereses propios de los estudiantes como base para la motivación y aprendizaje significativo; 4) la flexibilización del planteamiento didáctico según las circunstancias del propio proceso de investigación y el desarrollo de las circunstancias contextuales que envuelven el proceso educativo; 5) la coherencia entre el planteamiento didáctico y la evaluación. Esta debe ser necesariamente continua y formativa, basada en resultados y procesos y debe generar una retroalimentación igualmente continua y 6) el desarrollo de la conciencia en el alumnado de que es el principal responsable de sus logros y evaluación.

Por otro lado, la experiencia desarrollada no ha estado exenta de dificultades. En este sentido debiera de valorarse: 1) la dificultad de aplicar esta metodología en grupos grandes, sobre todo en lo relativo a la tutorización y evaluación, dadas las ratios de estudiantes por profesor en la universidad española; 2) el exceso de dedicación que supone este tipo de enseñanza para el profesorado y para el alumnado; 3) las dificultades que implica que todos los estudiantes acepten y se motiven en la realización del proyecto, dada la diversidad de situaciones personales y 4) lo complejo que es trasladar una evaluación basada en ocasiones en la narración a la calificación, cuando el sistema educativo ha entronizado la calificación "objetiva" como evidencia del logro.

Por último, la aplicación del ABPC en la asignatura de Técnicas Cualitativas nos ha permitido reflexionar sobre el incompleto encaje de las competencias específicas de la materia y de las generales y transversales del título con las metodologías innovadoras de enseñanza. Creemos que en general el título de Geografía y Ordenación del Territorio parece diseñado para ser impartido mediante planteamientos didácticos más tradicionales y esto conlleva que ciertas competencias como la creatividad, la capacidad de liderazgo, la mejora de la autoestima, etc. estén subrepresentadas. Estas competencias son esenciales en la educación universitaria actual y su inclusión en los planes y proyectos docentes tiene que ir a la par de la apuesta por la innovación metodológica.

\section{AGRADECIMIENTOS}

Los docentes quieren expresar su agradecimiento a los estudiantes por la colaboración en el trabajo de ABPC y a los agentes sociales que facilitaron el proceso investigación que estos desarrollaron.

\section{REFERENCIAS}

Barkley, E. F.; Cross, K.P. \& Major, C.H. (2007). Técnicas de aprendizaje colaborativo. Ministerio de Educación y Ciencia y Ediciones Morata, Madrid.

Bordas, M., \& Cabrera, F. (2001). Estrategias de evaluación de los aprendizajes centrados en el proceso. Revista Española de Pedagogía, 218, 25-48

Bould, D., Cohen, R., \& Sampson, J. (Eds.). (2013). Peer learning in higher education: Learning from and with each other. Routledge, London. https://doi.org/10.4324/9781315042565.

Bruffee, K.A. (1999). Collaborative learning: higher education, interdependence and the authority of Knowledge. John Hopkins University Press, Baltimore.

Cardozo, J. (2010). Los aprendizajes colaborativos como estrategia para los procesos de construcción de conocimiento. Revista Educación y Desarrollo Social, 4 (2), 87-102.

Delgado, K. (2016). Aprendizaje colaborativo. Cooperativa Editorial Magisterio, Bogotá.

Garrigós, J., \& Valero, M. (2012). Hablando sobre aprendizaje basado en proyectos con Júlia. REDU. Revista de Docencia Universitaria, 10 (3), 125-151. https://doi.org/10.4995/redu.2012.6017.

Guerra Santana, M., Rodríguez Pulido, J., \& Artiles Rodríguez, J. (2019). Aprendizaje colaborativo: experiencia innovadora en el alumnado universitario. Revista de Estudios y Experiencias en Educación, 18(36), 269-281. Retrieved from https://scielo.conicyt.cl/pdf/rexe/v18n36/0718-5162rexe-18-36-269.pdf.

Johnson, D., \& Johnson, R. (1999). Making cooperative learning work. Theory into Practice, 38 (2), 67-73.

Larmer, J., \& Mergendoller, J. R. (2010). 7 Essentials for Project-Based Learning. Educational Leadership, 68(1), 34-37

Maldonado Pérez, M. I. (2008). Aprendizaje basado en proyectos colaborativos. Una experiencia en educación superior. Laurus, 14 (28), 158-180. Retrieved from https://www.redalyc.org/articulo. oa?id=76111716009

Meneses Parra, R. D. (2013). Aprendizaje Basado en Proyectos (ABPr). Metodologías, Estrategias y Herramientas Didácticas para el diseño de cursos en ambientes virtuales de aprendizaje en la Universidad Nacional Abierta y a Distancia UNAD, 21. Retrieved from http://hdl.handle.net/10596/5396.

Monedero, C., \& Durán, D. (2002). Entramados: métodos de aprendizaje cooperativo y colaborativo. Edebe, Barcelona.

Pérez Gómez, A.I. (2012). Educarse en la era digital. Ediciones Morata, Madrid.

Travieso Valdés, D. \& Ortiz Cárdenas, T. (2018). Aprendizaje basado en problemas y enseñanza por proyectos: alternativas diferentes para enseñar. Revista Cubana de Educación Superior, 37(1), 124-133. Retrieved from http://scielo.sld.cu/scielo.php?script=sci_arttext\&pid=S0 257-43142018000100009\&lng=es\&tlng=es.

Vergara Ramírez, J.J. (2016). Aprendo porque quiero. El Aprendizaje Basado en Proyectos (ABP), paso a paso. SM, Madrid. 